Associate editor: P. Foster

\title{
Identification of therapeutic drug targets through genetically manipulated mice: Are we getting it right?
}

\author{
Klaus I. Matthaei* \\ Stem Cell E' Gene Targeting Laboratory, Structural Biology Program, The John Curtin School of Medical Research, ANU College of Medicine, Biology E Environment, \\ The Australian National University, Canberra, ACT 0200, Australia \\ Stem Cell Unit, Department of Anatomy, College of Medicine and King Khalid University Hospital, King Saud University, Riyadh 11461, Saudi Arabia
}

\section{A R T I C L E I N F O}

\section{Keywords:}

Mouse

Genetic manipulation

Transgenesis

Strain variation

Phenotype

\begin{abstract}
A B S T R A C T
A significant contribution to the identification of therapeutic targets in disease control and management has been made through the use of genetically modified mice in which specific gene function was altered. However, the genetic background of these mice can have a strong influence on the observed phenotype and, if not properly controlled, can lead to misinterpretation of data and incorrect conclusions as to the importance of particular genes in disease states. Nevertheless, it is becoming apparent that advantages may also ensue, since analysis of gene function in mixed (or a number of different) genetic backgrounds may more closely approximate the human genome and provide the potential to identify modifier genes and polymorphisms which influence the resulting phenotype of the target gene. A rigorous analysis of these strain-specific modifications of molecular function may thus afford a deeper understanding of the mechanisms underlying gene function and permit the development of more selective, specific and subtle molecular therapeutic approaches.
\end{abstract}

(C) 2009 Elsevier Inc. All rights reserved.

\section{Contents}

1. Introduction . . . . . . . . . . . . . . . . . . . . . . . . . . . . . . . . . . . . . . . . . 32

2. Modification of gene function in different genetic backgrounds . . . . . . . . . . . . . . . . . . . . . . 33

3. Identification of genetic polymorphisms through the use of different genetic backgrounds . . . . . . . . 33

4. Modification of gene function in mixed genetic backgrounds . . . . . . . . . . . . . . . . . . . . . . . . 34

5. Recent developments in generating genetically manipulated mice . . . . . . . . . . . . . . . . . . . 34

6. Conclusions ....................................... 35

Acknowledgments . . . . . . . . . . . . . . . . . . . . . . . . . . . . . . . . . . . . . 35

References . . . . . . . . . . . . . . . . . . . . . . . . . . 35

\section{Introduction}

The ability to delete or modify specific gene function in mice has had a huge influence on the identification of therapeutic targets for the control and management of a number of human diseases.

Abbreviations: BALB, BALB/c; B6, C57BL/6; COPD, chronic obstructive pulmonary disease; ES cells, embryonic stem cells; Gly, Glycine; GST, glutathione $S$ transferase; iPS, induced pluripotent stem; IL, interleukin; Ile, Isoleucine; Thr, Threonine; Val, Valine.

* Stem Cell \& Gene Targeting Laboratory, The John Curtin School of Medical Research, The Australian National University, Building 131, Garran Road Acton 0200, Australia. Tel.: +612 6125 3782; fax: +61261252499.

E-mail address: Klaus.Matthaei@anu.edu.au.
However, there are limitations of these techniques that provide the potential for the misinterpretation of data. In particular, variations in gene expression in different inbred mouse strains can have profound effects on the interpretation of gene function, if a given genetic manipulation is analysed in a mixed genetic background, rather than a pure inbred strain, and this is now considered a caveat of the procedure (Matthaei, 2004, 2007). For example, in the case of the p75 nerve growth factor deficient mice (Van der Zee et al., 1996), it was later found that the differences initially reported were most likely due to the strain differences of the mixed genetic background and not due to the gene modification itself, hence the subsequent retraction of the data (Hagg, 1999). However, strain variation in mice, if properly controlled, can also be used to our advantage since it provides the 
potential for the identification of molecular mechanisms through which gene function may be modified and hence the development of more specific and subtle molecular therapeutic approaches.

\section{Modification of gene function in different genetic backgrounds}

\section{1. $G_{\alpha} z$}

It was described earlier in a less accessible format (Matthaei, 2004) that functional deletion of the GTP binding protein $\mathrm{G}_{\alpha} \mathrm{z}$ in a C57BL/6/ $\mathrm{BALB} / \mathrm{c}$ (B6/BALB) F2 mixed genetic background resulted in $\mathrm{G}_{\alpha} \mathrm{z}^{-1-}$ mice that could raise their young normally (albeit that the offspring thrived less well than wild-type littermates). Surprisingly in a pure B6 background, offspring of homozygous $\mathrm{G}_{\alpha} \mathrm{z}^{-1-}$ mice died within $24 \mathrm{~h}$ of birth, whilst heterozygous $G_{\alpha} z^{+/-}$parents could rear their $G_{\alpha} z^{-/-}$ offspring normally. Importantly, when $\mathrm{B} 6 \mathrm{G}_{\alpha} \mathrm{z}^{-/-}$offspring were fostered to BALB $G_{\alpha} z^{+/+}$mothers, the offspring survived, whilst fostering of $G_{\alpha} z^{+/+}$offspring to $B 6 G_{\alpha} z^{-/-}$mothers again resulted in poor growth (Holgate, 2005). This indicated to us that there were genetic differences between the B6 and BALB mice, which, in the B6 strain, were responsible for the observed deficiency in maternal function of $G_{\alpha} z^{-/-}$females. Further investigation allowed us to determine that the $\mathrm{B} 6$ females were deficient in oxytocin secretion due to altered signalling via $G_{\alpha} \mathrm{z}$ and the $5 \mathrm{HT}-1 \mathrm{~A}$ receptor, a pathway known to regulate oxytocin levels and the milk letdown response (Holgate, 2005; Holgate et al., 2005).

In the case described above, the strain differences were highly informative and revealed that modifier genes are expressed in the BALB strain but not in the B6 strain, and these can compensate for a loss of $G_{\alpha} z$ function in certain tissues. The initial mixed genetic background therefore was beneficial for our studies and improved our understanding of the biological role of $G_{\alpha} z$. Indeed, if we had generated the mice in a pure $\mathrm{B} 6$ genetic background at the outset, we may never have discovered these modifications of $G_{\alpha} z$ because of the neonatal mortality of the $G_{\alpha} z$ deletion in the $B 6$ strain and consequent difficulty in studying tissue function.

\subsection{Interleukin-5}

The strain difference in the $G_{\alpha} z$ deficient mice was extremely dramatic, survival in the mixed background vs death in the B6 strain, and could hardly remain undetected once we had generated both strains of mice. It was also reported previously (Matthaei, 2007), and worth re-iterating here, that more subtle strain differences can be highly informative and contribute to a deeper understanding of the control of gene function.

Interleukin-(IL)-5 is a cytokine that regulates the differentiation and proliferation of blood immune cells called eosinophils (Campbell et al., 1988). These cells are part of the mammalian innate immune system and protect against parasites. However, there is also a correlation between the numbers of these cells present in the lungs of asthmatics and the severity of the disease, suggesting a causative role for these cells (Beasley et al., 1989; De Monchy et al., 1985) and a target for molecular therapeutics. In order to further investigate the role of IL-5 and eosinophils in the development of asthma we disrupted IL-5 function by gene targeting in B6 mice (Kopf et al., 1996). When tested in a mouse model of allergic lung disease, wild-type B6 mice developed all of the hallmark symptoms of human asthma, including blood eosinophilia, massive recruitment of eosinophils into the lung, eosinophil margination into the airway lumen, extensive lung damage and airways occlusion (Foster et al., 1996). Importantly, when treated with the spasmogen $\beta$-methacholine, mice also develop airways hyperreactivity similar to bronchospasm in humans. After deletion of IL- 5 function in the IL- 5 deficient mice, all of these features of allergic lung disease, including the airways hyperreactivity were completely absent (Foster et al., 1996). Moreover, when we infected the IL-5 deficient mice with a recombinant vaccinia virus expressing IL-5, the disease was fully reconstituted in the allergy model. We concluded from our studies that IL-5 is central to allergic lung disease and therefore may represent a promising therapeutic target for the treatment of asthma (Foster et al., 1996).

However, a contradictory finding was published at exactly the same time. Corry and colleagues, using a very similar allergy model, reported that although eosinophilia and lung damage were abolished by the inoculation of a monoclonal antibody against IL-5, the airways hyperreactivity remained (Corry et al., 1996). Of course, it could be argued that the use of a genetically deficient animal was a more efficient way of deleting gene function than the injection of neutralising antibodies, since the antibody may not have access to all relevant tissue locations. Again however, we noticed that there was a distinct strain difference between the two experiments. The IL-5 deficient mouse experiments were performed in B6 mice, whilst the antibody work was conducted in the BALB strain. We therefore crossed the IL- 5 deletion onto the BALB strain for 5 generations and subjected the N5.BALB IL-5 deficient mice to the allergy model. Indeed, our suspicions were correct and we were able to confirm the finding of Corry and colleagues. In the BALB strain, although eosinophil numbers decreased and lung damage remarkably improved, the airways hyperreactivity persisted in N5.BALB IL-5 deficient mice (Hogan et al., 1998). Interestingly, the data in the BALB mice but not the B6 mice were more representative of the human asthmatic condition. Administration of IL-5-specific monoclonal antibodies to humans dramatically reduced eosinophil numbers in the blood and sputum of patients for up to 4 months, however lung function was not improved (Leckie et al., 2000).

\section{Identification of genetic polymorphisms through the use of different genetic backgrounds}

\subsection{Interleukin-13}

The data described above alerted us to carefully analyse the genetic background in all of our experiments. By so doing, we were able to determine the cause of an apparent contradiction in data obtained with the IL-13 knockout mice (McKenzie et al., 1998). This mutation, when crossed from a 129/B6 mixed background to the BALB background for 5 generations (N5.BALB) still permitted airways hyperreactivity in our allergy model (Webb et al., 2004). However, after 7 crosses to BALB, it had been reported that the airways hyperreactivity was absent in N7.BALB IL-13 deficient mice (Walter et al., 2001). By careful analysis, we were able to show that this apparent discrepancy was due to a $\mathrm{Thr}^{49}$ Ile polymorphism (a threonine to isoleucine polymorphism at position 49) of the IL-4 receptor alpha (IL-4R $\alpha)$. This polymorphism is present as Thr in the 129 and B6 mice, and Ile in the BALB mice, and alters the signalling of IL-4 (Webb et al., 2004). Importantly, the N5.BALB mice still had the B6 isoform while the N7 had acquired the BALB isoform.

The discovery of the $\mathrm{Thr}^{49}$ Ile polymorphisms in the mouse strains has therapeutic implications for humans, since there are similar polymorphisms in the human IL-4R $\alpha$ gene that are suspected to correlate with human allergic disease (Webb et al., 2004) and may lead to differences in the expression of IgE, resulting in different allergic responses (Mitsuyasu et al., 1998; Mitsuyasu et al., 1999). Clearly, these differences between mouse strains and their counterparts in the human could allow a more in-depth understanding of allergic disease processes in humans.

\subsection{Glutathione S-transferase}

The glutathione S-transferase (GST) P1 gene in the human has a $\mathrm{Val}^{105}$ Ile polymorphism. The frequency of $\mathrm{Val}^{105} / \mathrm{Val}^{105}$ was found to be significantly lower in human asthmatics than in control subjects, 
where its presence conferred a sixfold lower risk of asthma than did the GSTP1 Ile $\mathrm{Il}^{105} / \mathrm{Ile}^{105}$ isoform (Fryer et al., 2000; Islam et al., 2009). Similar correlations may also exist between the GSTP1 polymorphism in humans and chronic obstructive pulmonary disease (COPD) (Cheng et al., 2004), cleft palate in offspring from parents that smoke (Krapels et al., 2008), as well as urinary tract carcinoma (Hsu et al., 2008). Interestingly, a comparable Val ${ }^{105} \mathrm{Gly}$ polymorphism in GSTP1/2 exists between B6 and BALB mice (Zhou et al., 2008). Moreover, there is a GSTP1/GSTP2 gene deficient mouse in which GSTP1/2 has been deleted in all tissues (Henderson et al., 1998). In these GSTP1/2 KO mice it would be possible to express different isoforms of GSTP1 (Val or Gly) under the control of tissue specific promoters in different genetic backgrounds (i.e. B6 or BALB) to determine the role of the two GSTP1 isoforms on the development of a specific disease such as COPD, or cleft palate. The combination of tissue specificity and genetic background should therefore be a powerful tool for the analysis of disease and lead to development of molecular therapeutics in these cases of known genetic polymorphisms.

\section{Modification of gene function in mixed genetic backgrounds}

While the development of genetically modified mice in a pure genetic background may allow the analysis of a single gene difference between the wild-type and the modified animals, a mixed genetic background at times also allows the analysis of a phenotype which is more physiologically relevant to humans. However which approach will be the most promising depends on the particular circumstances of each gene and may be hard to predict as shown below.

\subsection{Orexin}

Deletion of orexin containing neurons in orexin/ataxin-3 transgenic mice in a B6/DBA2 mixed genetic background results in a tendency for obesity with a marked increase in body weight from 14 weeks of age as compared to wild-type littermates, in spite of a reduction in food intake by $20 \%$ in the transgenic mice (Hara et al., 2005). Crossing of these mice to the $B 6$ background for 8 generations, however, completely abolished the difference in weight gain and also normalised the food intake (Hara et al., 2005). The data thus suggested that the B6 background was less sensitive to orexin deficiency than the DBA2 strain. However, challenge with a high-fat diet from 4 to 11 weeks of age did reveal a significant weight gain in the orexin/ataxin-3 mice, even in the N8.B6 background (Hara et al., 2005). This example demonstrates the potential advantage of a mixed genetic background since the relationship between orexin and weight gain may not have been discovered, had these mice been generated in a single strain. In this case crossing the deletion onto a pure genetic background resulted in a masking of the gene function.

\subsection{Sox 18}

In contrast to the case above, a pure genetic background may be the most informative. The ragged $(R a)$ mutation is a natural mutant that arose spontaneously in a crossbred stock of mice [see (Pennisi, $2000 \mathrm{~b})]$. Ra heterozygotes $(R a /+)$ are viable and healthy but have modified coats with modified hair structure, whilst $R a / R a$ homozygotes lack whiskers and coat hair and rarely survive past weaning. In a phenotypically more severe form of ragged, called opossum, the heterozygotes $R a^{o p} /+$ are similar to homozygous $R a / R a$ mice and homozygotes $R a^{o p} / R a^{o p}$ die at 11.5 days post-coitum. Recently, the gene for $R a$ (Sox18) was cloned and subsequently deleted in mice (Pennisi, Bowles, Nagy, Muscat, \& Koopman, 2000). Surprisingly, Sox $18^{-1-}$ mice were found to be viable and fertile. They have no obvious cardiovascular defects and only display a mild coat defect, i.e. having a reduced proportion of "zigzag" hairs. It was hence concluded, "this study provides the first evidence of a SOX gene that is largely dispensable for embryonic development" (Pennisi et al., 2000b). However, the mice were generated in a complex 129/CD1 F2 genetic background consisting of three mouse strains. The R1 ES cells that were used for the homologous recombination had been previously isolated from an F1 mouse embryo derived from an intercross of $129 X 1 /$ sv and $129 S 1 /$ sv mice (Nagy et al., 1993). The R1 chimæra were subsequently bred with CD1 mice to observe germline transmission and the mice analysed in this R1/CD1 mixed background (25\% 129SvX1/sv: 25\% 129S1/sv: 50\% CD1) showed no phenotype (Pennisi et al., 2000a). However, when the Sox18 mutation was crossed from the 129/CD1 background to the B6 genetic background for 11 generations, a clear phenotype became obvious (Francois et al., 2008). The N11.B6 Sox $18^{-/-}$mice exhibited gross subcutaneous oedema and foetal lethality as homozygotes, and mild cutaneous oedema as heterozygotes whereas no phenotype had been previously observed in the mixed genetic background. In this case, the mixed genetic background had robustly disguised the Sox18 deletion phenotype and crossing to a purer genetic background increased the severity and phenotypic expression of the genetic manipulation.

\section{Recent developments in generating genetically manipulated mice}

\subsection{Gene deficient mice}

Described above are some strain differences that have led to a better understanding of human disease and there are many more examples in the literature. It is also clear that, to obtain the most information, several inbred genetic backgrounds as well as mixed genetic backgrounds may have to be studied. However, which genetic strain is most closely related to the human and is therefore best to model human disease and treatment? It is of course nonsense to believe that the homogeneous genetic makeup of a given inbred mouse strain can be used to model the wide genetic diversity that is seen in human populations. On the other hand, as discussed above, there are some polymorphisms in particular mouse strains that are highly informative. Clearly, a wide range of different mouse strains should be analysed to ensure that the full spectrum of biological function of the target gene is revealed. However, the ability to generate genetically manipulated mice in a wide range of strains has been previously limited by the availability of embryonic stem (ES) cells from only the more common strains, such as B6, BALB, 129, DBA, CBA [see e.g. Ledermann (2000)], but not the more exotic strains such as the nonobese diabetic (NOD) strain [unless F1 offspring are used (Brook et al., 2003)].

Recent advances have altered this situation as it is now possible to generate induced pluripotent stem (iPS) cells from mouse (and human) by introducing defined factors like Oct3/4, Sox2, c-Myc and KLf4 into fibroblasts using retrovirus or lentivirus transfection (Takahashi \& Yamanaka, 2006). Indeed the iPS cells have the ability to contribute to the germline when injected into blastocyst stage embryos, in a manner similar to ES cells, and hence produce iPS derived mouse strains (Takahashi \& Yamanaka, 2006). However, the use of viruses to generate these cells carries the risk of transforming the cells to become tumourigenic since the viral DNA can integrate into the genome. More recently therefore, iPS cells with germline capacity were generated without the use of viral vectors circumventing this potential problem (Okita et al., 2008).

Of interest here is that multipotent cells have also been isolated from the olfactory mucosa that can differentiate into many different cell types in vitro (Feron et al., 1999) and incorporate into many tissues when transplanted into the chick embryo (Murrell et al., 2005). Importantly, these olfactory stem cells are multipotent without the need for genetic manipulation and do not produce tumours or teratomas when transplanted (Murrell et al., 2008), yet may have the potential to contribute to the germline if they were to be injected into blastocysts. 
Although to date no homologous recombination has been performed in iPS or olfactory cells it may be possible to do this in the near future. These two new sources of pluripotent cells may therefore allow for the first time the generation of genetically deficient mice in all available genetic backgrounds.

\subsection{Transgenic mice}

To date, the injection of DNA constructs directly into the pronucleus of a single-cell embryo is still the most common method for generating transgenic mice. However, some strains are refractory to this method, because it is impossible to visualise the pronucleus in those embryos. Recently, a method was developed with which transgenic mice can be generated by electroporating the recombinant DNA directly into the cells of the testis (Dhup \& Majumdar, 2008). After a period of recovery, these animals produce transgenic founder offspring from which new strains can be established by breeding. This method should potentially allow the generation of transgenic mice (and those from other species such as rats or guinea pigs) from all strains, thereby also improving our ability to carefully analyse the phenotype of the overexpression (as opposed to gene deletion described above) of a particular gene of interest in a variety of suitable genetic backgrounds.

\section{Conclusions}

Molecular therapeutics relies on the ability of a drug to appropriately modify its target for the successful treatment of disease. Genetic variation in the human population however often changes the efficacy of the drug and can lead to ineffectual treatment. Multidrug resistance of certain cancers may be an example of this. Fortunately, as described here, the genetic background of mice in which specific gene expression is tested can have a strong influence on the observed phenotype and, if not carefully controlled, can lead to misinterpretation of data and incorrect conclusions. However, the exact same strain variation can also be used to our advantage. Of course it is naïve to think that any particular mouse strain will be fully representative of the complex human gene pool. However, the development of the ability to manipulate the germline of mice in previously unusable strains by homologous recombination in iPS cells, or by transgenesis using testis electroporation, should permit a far better understanding of gene function in human health and disease. Importantly for the pharmaceutical industry, the isolation of pluripotent stem cells from genetically diverse strains of mice will enable high-throughput drug screening programmes to be applied to a broader genetic pool. Further down the track but increasingly relevant, the development of pluripotent cell lines from humans will enable disease specific or patient specific cells to be available for similar molecular therapeutics and verification of data obtained in mice. Taken together, it is clear that strain variation in mice is proving to be a benefit that may permit a more in-depth understanding of gene function and thereby the potential for the development of more specific and subtle molecular therapeutic approaches for the treatment of human disease.

\section{Acknowledgments}

I am very grateful to the many colleagues who have helped me with in-depth discussions about this topic and who are referenced throughout this publication. In addition I would specifically like to thank Professor Caryl Hill and Dr. Michael Frese for critical reading of this manuscript. I would also like to acknowledge support from NHMRC grants \#418071, \#471421 and \#471424 and an ACT Health \& Medical Research Support Grant.

\section{References}

Beasley, R., Roche, W. R., Roberts, J. A., \& Holgate, S. T. (1989). Cellular events in the bronchi in mild asthma and after bronchial provocation. Am Rev Respir Dis 139(3), 806-817.

Brook, F. A., Evans, E. P., Lord, C. J., Lyons, P. A., Rainbow, D. B., Howlett, S. K., et al. (2003). The derivation of highly germline-competent embryonic stem cells containing NOD-derived genome. Diabetes 52(1), 205-208.

Campbell, H. D., Sanderson, C. J., Wang, Y., Hort, Y., Martinson, M. E., Tucker, W. Q., et al. (1988). Isolation, structure and expression of cDNA and genomic clones for murine eosinophil differentiation factor. Comparison with other eosinophilopoietic lymphokines and identity with interleukin-5. Eur J Biochem 174(2), 345-352.

Cheng, S. L., Yu, C. J., Chen, C. J., \& Yang, P. C. (2004). Genetic polymorphism of epoxide hydrolase and glutathione S-transferase in COPD. Eur Respir J 23(6), 818-824.

Corry, D. B., Folkesson, H. G., Warnock, M. L., Erle, D. J., Matthay, M. A., Wiener-Kronish, J. P., et al. (1996). Interleukin 4, but not interleukin 5 or eosinophils, is required in a murine model of acute airway hyperreactivity. J Exp Med 183(1), 109-117.

De Monchy, J. G., Kauffman, H. F., Venge, P., Koeter, G. H., Jansen, H. M., Sluiter, H. J., et al. (1985). Bronchoalveolar eosinophilia during allergen-induced late asthmatic reactions. Am Rev Respir Dis 131(3), 373-376.

Dhup, S., \& Majumdar, S. S. (2008). Transgenesis via permanent integration of genes in repopulating spermatogonial cells in vivo. Nat Methods 5(7), 601-603.

Feron, F., Mackay-Sim, A., Andrieu, J. L., Matthaei, K. I., Holley, A., \& Sicard, G. (1999). Stress induces neurogenesis in non-neuronal cell cultures of adult olfactory epithelium. Neuroscience 88(2), 571-583.

Foster, P. S., Hogan, S. P., Ramsay, A. J., Matthaei, K. I., \& Young, I. G. (1996). Interleukin 5 deficiency abolishes eosinophilia, airways hyperreactivity, and lung damage in a mouse asthma model. J Exp Med 183(1), 195-201.

Francois, M., Caprini, A., Hosking, B., Orsenigo, F., Wilhelm, D., Browne, C., et al. (2008). Sox18 induces development of the lymphatic vasculature in mice. Nature 456 (7222), 643-647.

Fryer, A. A., Bianco, A., Hepple, M., Jones, P. W., Strange, R. C., \& Spiteri, M. A. (2000). Polymorphism at the glutathione S-transferase GSTP1 locus. A new marker for bronchial hyperresponsiveness and asthma. Am J Respir Crit Care Med 161(5), 1437-1442.

Hagg, T. (1999). Neuronal cell death: Retraction. Science 285(5426), 340

Hara, J., Yanagisawa, M., \& Sakurai, T. (2005). Difference in obesity phenotype between orexin-knockout mice and orexin neuron-deficient mice with same genetic background and environmental conditions. Neurosci Lett 380(3), 239-242.

Henderson, C. J., Smith, A. G., Ure, J., Brown, K., Bacon, E. J., \& Wolf, C. R. (1998). Increased skin tumorigenesis in mice lacking pi class glutathione S-transferases. Proc Natl Acad Sci U S A 95(9), 5275-5280.

Hogan, S. P., Matthaei, K. I., Young, J. M., Koskinen, A., Young, I. G., \& Foster, P. S. (1998). A novel T cell-regulated mechanism modulating allergen-induced airways hyperreactivity in BALB/c mice independently of IL-4 and IL-5. J Immunol 161(3), 1501 - 1509.

Holgate, J. (2005). Investigation of the neonatal mortality phenotype of GaZ knockout mice: A role for $\mathrm{Gz}$ in the oxytocin pathway. BSc. Honours Thesis, The Australian National University, Canberra Australia.

Holgate, J., Megirian, D., Matthaei, K. I., \& Hendry, I. A. (2005). Examination of oxytocin levels in BALB/C and C57BL/6 GalphaZ knockout mice: A role for GalphaZ in oxytocin release. Annual Meeting of the Society for Neuroscience, Perth Australia.

Hsu, L. I., Chiu, A. W., Huan, S. K., Chen, C. L., Wang, Y. H., Hsieh, F. I., et al. (2008). SNPs of GSTM1, T1, P1, epoxide hydrolase and DNA repair enzyme XRCC1 and risk of urinary transitional cell carcinoma in southwestern Taiwan. Toxicol Appl Pharmacol 228(2), 144-155.

Islam, T., Berhane, K., McConnell, R., Gauderman, W. J., Avol, E., Peters, J. M., et al. (2009). Glutathione-S-transferase (GST) P1, GSTM1, exercise, ozone and asthma incidence in school children. Thorax 64(3), 197-202.

Kopf, M. Brombacher, F, Hodgkin, P. D. Ramsay, A. J. Milbourne, E. A, Dai, W. J. et al (1996). IL-5-deficient mice have a developmental defect in CD5 + B-1 cells and lack eosinophilia but have normal antibody and cytotoxic T cell responses. Immunity 4(1), $15-24$.

Krapels, I. P., Raijmakers-Eichhorn, J., Peters, W. H., Roelofs, H. M., Ras, F., \& SteegersTheunissen, R. P. (2008). The I,105V polymorphism in glutathione S-transferase P1, parental smoking and the risk for nonsyndromic cleft lip with or without cleft palate. Eur J Hum Genet 16(3), 358-366.

Leckie, M. J., ten Brinke, A., Khan, J., Diamant, Z., O'Connor, B. J., Walls, C. M., et al. (2000). Effects of an interleukin-5 blocking monoclonal antibody on eosinophils, airway hyperresponsiveness, and the late asthmatic response Lancet 356(9248), 2144-2148.

Ledermann, B. (2000). Embryonic stem cells and gene targeting. Exp Physiol 85(6), $603-613$.

Matthaei, K. I. (2004). Caveats of gene targeted and transgenic mice. In R. Lanza, J. Gearhart, B. Hogan, D. W. Melton, R. Pederson, J. Thomson, \& W. West (Eds.), Handbook of Stem Cells, Embryonic Stem Cells vol. 1. (pp. 589-598) Elsevier: Academic Press.

Matthaei, K. I. (2007). Genetically manipulated mice: A powerful tool with unsuspected caveats. J Physiol 582(Pt 2), 481-488.

McKenzie, G. J., Emson, C. L., Bell, S. E., Anderson, S., Fallon, P., Zurawski, G., et al. (1998). Impaired development of Th2 cells in IL-13-deficient mice. Immunity 9(3), 423-432.

Mitsuyasu, H Izuhara, K, Mao, X Q Gao, P. S, Arinobu, Y Enomoto, T et al (1998). Ile50Val variant of IL4R alpha upregulates IgE synthesis and associates with atopic asthma. Nat Genet 19(2), 119-120.

Mitsuyasu, H., Yanagihara, Y, Mao, X. O, Gao, P. S., Arinobu, Y, Ihara, K, et al. (1999). Cutting edge: Dominant effect of Ile50Val variant of the human IL-4 receptor alphachain in IgE synthesis. J Immunol 162(3), 1227-1231.

Murrell, W., Feron, F., Wetzig, A., Cameron, N., Splatt, K., Bellette, B., et al. (2005). Multipotent stem cells from adult olfactory mucosa. Dev Dyn 233(2), 496-515. 
Murrell, W., Wetzig, A., Donnellan, M., Feron, F., Burne, T., Meedeniya, A., et al. (2008). Olfactory mucosa is a potential source for autologous stem cell therapy for Parkinson's disease. Stem Cells 26(8), 2183-2192.

Nagy, A., Rossant, J., Nagy, R., Abramow-Newerly, W., \& Roder, J. C. (1993). Derivation of completely cell culture-derived mice from early-passage embryonic stem cells. Proc Natl Acad Sci U S A 90(18), 8424-8428.

Okita, K., Nakagawa, M., Hyenjong, H., Ichisaka, T., \& Yamanaka, S. (2008). Generation of mouse induced pluripotent stem cells without viral vectors. Science 322(5903), 949-953.

Pennisi, D., Bowles, J., Nagy, A., Muscat, G., \& Koopman, P. (2000). Mice null for sox18 are viable and display a mild coat defect. Mol Cell Biol 20(24), 9331-9336.

Pennisi, D., Gardner, J., Chambers, D., Hosking, B., Peters, J., Muscat, G., et al. (2000). Mutations in Sox18 underlie cardiovascular and hair follicle defects in ragged mice. Nat Genet 24(4), 434-437.
Takahashi, K., \& Yamanaka, S. (2006). Induction of pluripotent stem cells from mouse embryonic and adult fibroblast cultures by defined factors. Cell 126(4), 663-676.

Van der Zee, C. E., Ross, G. M., Riopelle, R. J., \& Hagg, T. (1996). Survival of cholinergic forebrain neurons in developing p75NGFR-deficient mice. Science 274(5293), $1729-1732$.

Walter, D. M., McIntire, J. J., Berry, G., McKenzie, A. N., Donaldson, D. D., DeKruyff, R. H., et al. (2001). Critical role for IL-13 in the development of allergen-induced airway hyperreactivity. J Immunol 167(8), 4668-4675.

Webb, D. C., Matthaei, K. I., Cai, Y., McKenzie, A. N., \& Foster, P. S. (2004). Polymorphisms in IL-4R alpha correlate with airways hyperreactivity, eosinophilia, and Ym protein expression in allergic IL-13-/ - mice. J Immunol 172(2), 1092-1098.

Zhou, J., Wolf, C. R., Henderson, C. J., Cai, Y., Board, P. G., Foster, P. S., et al. (2008) Glutathione transferase p1: An endogenous inhibitor of allergic responses in a mouse model of asthma. Am J Respir Crit Care Med 178(12), 1202-1210. 